\title{
Morphometric versus densitometric assessment of coronary vasomotor tone-an overview
}

\author{
P.-A. Doriot, N. Guggenheim, P.-A. Dorsaz and W. Rutishauser \\ Cardiology Center, University Hospital, Geneva, Switzerland
}

KEY WORDS: Coronary vasomotor tone, coronary morphometry, coronary densitometry.

The main advantage of the morphometric approach is that the spatial orientation of the vessel with respect to the image intensifier is not very important. Its most severe limitations are that reasonable accuracy can only be obtained with circular lumina, and that accuracy decreases rapidly with the vessel diameter. The densitometric approach is much less dependent on the shape of the lumen and on the correct identification of the vessel wall in the image. A further essential advantage is that one measures directly the cross-sectional area of the vessel instead of a 'diameter' of low haemodynamic relevance. Severe requirements must however be met if the potential accuracy of densitometry is to be fully exploited. The morphometric approach seems thus preferable for absolute or relative diameter measurements on intact vessels, while densitometry is superior in case of irregular or small lumina. Morphometric calibration using the injection catheter can induce non-negligible errors in both approaches. Grid calibration is probably more accurate, but also more tedious. In the densitometric approach, '3D-calibration' by help of a cube of known size allows also determination of the spatial orientation of the vessel in space. This solution requires however biplane imaging.

\section{Introduction}

The importance of accurate measurements of coronary vasomotor tone is nowadays well acknowledged. Visual estimation of arterial calibres in angiographic images is increasingly replaced by computer-assisted methods. Computer assistance provides precision (reproducibility). Accuracy however depends on a lot of factors, some of which are controllable and others not. In the following, we examine the problems of the morphometric and densitometric approaches (measurement of diameters versus measurement of cross-sections).

\section{The morphometric approach}

Coronary angiography being a perspective projection of vessels onto a curved screen, the diameters measured in the image depend on the spatial location and, to a lesser extent, on the orientation of the considered cross-sections with respect to the angiocardiographic unit. When

Address for correspondence: P.-A. Doriot, Cardiology Center, University Hospital, CH-1211 Geneva 4, Switzerland. pin-cushion distortion is negligible, only the projections onto the central X-ray beam element of the two spatial distances 'focus/cross-section' and 'focus/catheter segment selected for calibration' must be taken into account. Neglecting an eventual difference between these two distances can result in a diameter error of several percent. In the following, projections of distances 'focus/object' onto the central beam element are called 'depths', 'profiles' are always intensity profiles obtained by measuring the grey levels across the vessel image, the 'screen' is always the entry screen of the image intensifier (I.I.) and the 'focus' the X-ray focus.

Pin-cushion distortion induces a broadening of the vessel. It is composed of a radial and of a tangential component, both defined with respect to the screen centre and expressed in percent. Both components increase radially and depend further on the size and magnification mode of the I.I., and on the distance 'focus/screen center'. Since they are not identical, vessel broadening depends on the actual orientation of the vessel in the image with respect to the screen centre. It is maximal when the diameter is oriented radially, and minimal when it is 
oriented tangentially. The difference increases radially and can attain several percent.

Whether pin-cushion distortion should be corrected or not depends on its cumulated effect with eventual depth differences, and on the kind and goal of the measurement. In routine quantification of stenosis, neither pin-cushion distortion nor depth differences will impair appreciably the accuracy of the obtained narrowing percentage and residual cross-section, because of the much greater error resulting from the irregular shape of the stenotic lumen. Correction of pin-cushion distortion and of depth differences seems however indicated in assessing small evolutive changes of stenotic lumina, because the precision (reproducibility) of stenosis measurements appeared to be very high $^{[1]}$ and since the geometric parameters are likely to vary in repeated catheterism. For absolute diameter measurements of intact arterial segments, both corrections are also indicated, provided that the 'quality' of the profile is accordingly high (which is usually not the case for small arteries).

All the geometric errors being rectifiable, diameters of coronary arteries with circular cross-sections could be determined very accurately if image noise and point-spread transfer function would not make real profiles have no clearly identifiable base points. Because of the variability of the transfer function, of the magnitude of the noise and of the irregularity of luminal shapes, deconvolution of the profiles aiming at the restoration of the base points is not helpful. Diameters must thus be determined from two other 'appropriate' profile points, that means from points standing in a sufficiently tight relationship with the true arterial diameter. The location of such appropriate profile points depends however: (1) on the true diameter; (2) on the iodine concentration inside the vessel; (3) on the degree of homogeneity of the iodine concentration; (4) on the relationship between thickness of contrast medium and resulting profile amplitude; (5) on the nature of the 'underlying background' (myocardium, lung or else; homogeneous or not; flat or 'slopy'); (6) on the noise. Some of these factors (i.e. 1, 2 and 4) can be taken into account using confirmed experimental results.

Due to the point-spread transfer function, profiles of circular arteries will usually have two inflexion points, to which correspond two extrema of the first 'derivative' of the profile 'function'. Extrema of the second derivative can be used in turn to define two profile 'base points'. Whether inflexion points, base points or a combination of both are more appropriate for diameter measurements than, for instance, the profile width at half-height has to be assessed experimentally. Thus, even in the case of circular lumina, the variability of the transfer function and the cited factors 1 to 6 will limit accuracy to at least some diameter percentage.

Computer assistance implies digitization of the images at some stage of the method. Since the digitization steps for coordinates and grey levels can seldom be selected optimally with respect to the spatial and contrast resolutions of the imaging process, digitization results in a more or less coarse discretization of the values and in digitization noise. The impact of digitization coarseness has been demonstrated by Sandor $e t$ al. ${ }^{[2]}$ who used digitally acquired, unsubtracted $512 \times 512 \times 8$ bits images to measure the diameters of 'phantom' arteries with circular cross-section, filled with Renografin $76 \%$ and immersed in water. They found that true and obtained diameters were more or less linearly related only from $3 \mathrm{~mm}$ upwards, and that edge detection (inflexion points) was not reproducible below $1.54 \mathrm{~mm}$. In digitally acquired images, direction and unit of coordinates as well as grey level steps are definitively fixed. Since the vessel axis will usually not be parallel to the $x$ - or $y$-axis of the images, new profiles must be computed by two-dimensional interpolation of coordinates and grey levels, whereby the interpolation scheme must preserve best the spare information available. Interpolation allows work at subpixel levels, which is mandatory, but does not create additional information. Thus, it does not solve the problem of fitting a profile curve through to few original points. Noise makes data averaging necessary ${ }^{[2]}$. Averaging of only grey levels, as is often done, can induce errors. For instance, averaging two or more frames at the same phase of different cardiac cycles will result in a too broad average diameter due to the imperfect periodicity of the heart beat. Broadening can also occur in averaging adjacent profiles in the same image. Since subsequent profile smoothing or curve fitting do not compensate for this effect, the individual profiles should be repositioned against each other before grey level averaging, for 
instance by cross-correlation. An alternative is to determine the diameters individually and to average them. Discretization, digitization noise and noise of the native image make profile smoothing or the fitting of analytical functions necessary. Which type of curve ( $n$th order polynomial, Fourier series, cubic splines or else) and which profile parts should be fitted ('left' and 'right' profile parts separately or both together, left and right limits of the intervals) are further questions which have not yet received definitive answers. The worth of each method must ultimately be assessed by the experimental demonstration of its accuracy. Spears et al. ${ }^{[3]}$ compared three edge detection methods on cylindrical 'phantom' arteries immersed in water, imaged with $35 \mathrm{~mm}$ cinefilm and calibrated with the help of a grid. The correct diameter appeared to lie between inflexion and base points. They also overlaid the phantom arteries onto the chest of patients and obtained $\pm 0 \cdot 10 \mathrm{~mm}$ diameter accuracy with the inflexion point method. The base point method was less accurate $( \pm 0.14 \mathrm{~mm})$, which is in contradiction with the results of Siebes et al..$^{[4]}$ who used the injection catheter for calibration.

If the lumen is irregularly shaped, the relation between inflexion points, base points or other points with any true dimensions of the cross-section is indeterminable. Rosenberg et al. ${ }^{[5]}$ compared in 20 post-mortem coronary segments the stenotic diameter obtained angiographically (monoplane) to the true minimum, and to the true maximum stenotic diameter determined subsequently by microscope examination. Although they used mask subtracted images and contrast medium injections into the artificially perfused segments, their experimental conditions were more favourable than in real coronary angiography since the background was homogeneous (water). If one eliminates the four segments with true minimum diameter smaller than $1.0 \mathrm{~mm}$ from their data, the diameters obtained from their better method (method 2) spread from $70 \%$ underestimation to $66 \%$ overestimation (mean $=-5.8 \%$ ) of the true average stenotic diameter (calculated as square root of the product 'true minimum diameter' times 'true maximum diameter'). Thus, the accuracy of the morphometric approach is very low in case of irregularly shaped lumen. Consequently, the usefulness of corrections for depth differences and pin-cushion distortions must really be examined with respect to the vessels to be studied and to the particular goal of the measurements.

\section{The densitometric approach}

The densitometric approach eliminates the problems of precise wall identification and of irregular luminal shape. Wall identification is not critical because the bulk of the intensity profile contributes more strongly to the determination of the cross-sectional area than the side parts. Insensitivity to the particular luminal shape is not always perfect but techniques allowing solution of occasional problems exist. A further advantage is that one measures directly the luminal area instead of one or two functionally much less relevant 'diameters'. Finally, in order to obtain an arterial crosssection with $20 \%$ accuracy, the average stenotic diameter obtained morphometrically should be accurate to about $10 \%$, which is far from what can be expected. Thus, from this methodological point of view, the densitometric approach appears very promising.

Perfect insensitivity to the luminal shape requires proportionality between chord length inside the artery and associated intensity value of the profile. In comparative measurements on phantom 'arteries' of increasing diameter, filled with contrast medium of $370 \mathrm{mg} \mathrm{ml}^{-1}$ iodine concentration, oriented parallel to the screen of the I.I., and imaged at $60 \mathrm{kV}$, one can however observe that with increasing diameter the increase in profile height weakens more and more ${ }^{[6]}$. Non-linearity and consequently shape dependence increase with the product 'thickness $X$ iodine concentration of contrast medium', and decrease with increasing $X$-ray tube voltage $(\mathrm{kV})$. Shape dependence is automatically accompanied by a dependence on the rotational orientation of the lumen (that is, around the vessel axis) with respect to the $\mathrm{X}$-rays. Fortunately, both effects do not impinge severely (a few percent) onto the accuracy of relative measurements of cross-sections imaged with the same parameters. ${ }^{[71}$ However, if one measures the degree of stenosis densitometrically, calibrates then morphometrically the intact proximal lumen and uses its area together with the measured degree of stenosis to obtain the true area of the stenotic cross-section, the resulting error may be greater (between 
$-8 \%$ and $+45 \%$ in the case of a $5 \mathrm{~mm}$ artery $)^{|7|}$. The problem of non-linearity can be attenuated by strong copper filtration of the X-ray beam, use of high $\mathrm{kV}$ and low iodine concentrations ${ }^{|8,9|}$. Image contrast and signal-to-noise ratio will however decrease. Non-linearity should also be kept in mind for following reason: since arteries cannot always be oriented parallel to the screen of the I.I., the thickness of contrast medium encountered locally by the X-rays is usually greater than for parallel orientation. For instance, in a $3.5 \mathrm{~mm}$ artery oriented at 45 degrees, the actual deviation of linearity would be the same as in a $5 \mathrm{~mm}$ artery oriented parallel.

The native profiles can be corrected pixel by pixel for non-linearity in order to obtain fictive profiles proportional to the corresponding thicknesses of contrast medium inside the artery ${ }^{(6)}$. The orientation of the vessel in space and the actual shape of the lumen are thereby meaningless, but the actual $\mathrm{kV}$ and the iodine concentration in the vessel during acquisition of the relevant images must be known. Thus, momentary total filling of the arterial segment of interest with contrast medium must be achieved, nevertheless, this is a basic requirement of the densitometric approach. The areas obtained with or without preliminary correction of non-linearity should also be corrected for non-parallelism with the screen if the artery was angled more than \pm 15 degrees (which corresponds to an error of $\pm 3 \%$ ). An accurate way to determine the actual angle is $3 \mathrm{D}$ reconstruction ${ }^{\{10\}}$. A future alternative will be to determine and to select projections in which the arterial segment of interest is parallel to the screen $^{[11]}$. This will however restrict the number of measurable stenoses in a particular projection view.

The geometric aspects discussed in the first section also play a role. The intensity profile of an artery located at $70 \mathrm{~cm}$ from the focus is $5 \%$ broader than when the artery is located at $73.5 \mathrm{~cm}$ but its height remains constant (at least if pin-cushion distortion is negligible). Therefore, the area under an intensity profile depends on the depth of the considered cross-section in the same manner as the apparent diameter. Not yet assessed is how far profile widening resulting from pin-cushion distortion is compensated by a decrease of the profile height. Also insufficiently assessed is how accurately the computed areas do reflect the true cross-sectional areas. Besides the problems cited above, one has still to investigate, particularly for small arterial crosssections, the impact of the sources of statistical fluctuations such as local iodine concentration, $\mathrm{X}$-ray emission and attenuation, $\mathrm{X}$-ray and other conversions in the 1.1., photographic imaging in case of cinefilm, structural noise, superimposition with spurious contrast medium in the myocardium, calcifications, etc.

Digitization of coordinates and grey levels is of course necessary. The associated problems are basically the same as in the morphometric approach but curve fitting is not necessary.

\section{Calibration problems}

In the morphometric approach, calibration by help of the injection catheter can induce non-negligible errors even when pin-cushion distortion and depth differences between catheter and considered cross-sections are compensated. Siebes et al. ${ }^{[4]}$ showed that the obtained calibration factor depends on the catheter manufacturer, on the iodine concentration in the catheter and on the particular edge detection technique used. They also pointed out that it may depend further on the type of catheter (wire-braided or not), and that the true diameter of the latter can differ appreciably from its nominal value. Using $8 \mathrm{~F}$ catheters from two manufacturers, filled with Renografin $76 \%$ and immersed in $10 \mathrm{~cm}$ water, they observed up to $17 \%$ diameter overestimation on a cylindrical $3 \mathrm{~mm}$ 'phantom' artery. Catheter wall and 'arterial' wall were defined either by the inflexion points or by the base points. The influence of the cited factors was found smaller using the base points.

Spears et al. ${ }^{|3|}$ found on the contrary no difference in accuracy between inflexion and base points methods on cylindrical 'phantom' arteries immersed in water, and a greater degradation of the base points method when the patient chest was used as background. A further apparent contradiction with the results of Siebes et al. is that increasing the iodine concentration resulted in an underestimation of the obtained diameter instead of an overestimation. This is probably due to the fact that Spears et al. used a grid for calibration.

In the study of Rosenberg et al. ${ }^{[5]}$, method 1 was found to overestimate considerably the true diameter. However, the authors used in method 1 a steel ball $(6 \mathrm{~mm})$ for calibration, and in 
method 2, a vein graft cannula $(1.5 \mathrm{~mm})$, whereby the diameter of the ball in the image was determined subjectively. This could explain the discrepancy between method 1 and 2 .

Thus, diameter calibrations by help of a grid, of the injection catheter or of a small steel ball are not equivalent. Calibration by use of the injection catheter is practical, especially if one ignores depth differences. Accuracy is however compromised by the above effects. Use of a grid eliminates all of the problems except the dependence on actual iodine concentration in the artery. The depth difference between grid and the considered arterial cross-section must, however, be taken into account. A further alternative is $3 \mathrm{D}$-reconstruction ${ }^{[10]}$ since this approach also allows for calibration.

In the densitometric approach, morphometric calibration requires a circular and measurable cross-section at some place on the vessel image so the previous considerations are also relevant. One can also calibrate densitometrically using the profile area of a calibration tube with translucent wall, filled with contrast medium of the same concentration as that in the vessel and placed onto the chest of the patient. This requires however biplane imaging in order to take the depth difference between artery and calibration tube into account. Densitometric calibration by use of the injection catheter is not accurate because of the influence of the catheter wall onto the profile area and due to the small size of the catheter lumen.

\section{Conclusion}

The main advantage of the morphometric approach is that the spatial orientation of the vessel needs not be determined. Its most severe limitation is that the area of irregularly shaped or small lumina cannot be measured accurately. In the densitometric approach, identification of the vessel wall and shape of the lumen are not critical. Moreover, one measures directly the luminal area instead of a functionally irrelevant 'diameter'. Densitometry is therefore potentially more accurate. However, the requirements of densitometry are still difficult to fulfil (orientation of the vessel parallel to the screen of the image intensifier or subsequent determination of the former by 3D-reconstruction, no superimposition with other opacified structures, momentary total filling of the vessel, correction of non-linearity if necessary, problem of cinefilm characteristics). Thus, the morphometric approach still seems preferable for calibre measurements of major, intact coronary arteries. In the case of irregular or small lumina, accuracy can only be expected from the densitometric approach.

\section{References}

[1] Reiber JHC, Kooijman CJ, Slager CJ et al. Coronary artery dimensions from cineangiogramsMethodology and validation of a computer-assisted procedure. IEEE Transactions on Medical Imaging 1983; Vol. MI-3: No. 3, 131-41.

[2] Sandor T, Spears JR, Boxt LM et al. Utilization of the Monte Carlo technique to assess precision in the measurement of coronary arteries. SPIE Vol. 555 Med Imag Instrumentation 1985; 85: 159-64.

[3] Spears JR, Sandor T, Als AV et al. Computerized image analysis for quantitative measurement of vessel diameter from cineanigograms. Circulation 1983; 68: 453-61.

[4] Siebes M, Selzer RH. How accurate is the catheter as a reference for arterial dimensions in quantitative coronary angiography? In: Computers in Cardiology 85, IEEE Computer Society Press, 1985: 9-14.

[5] Rosenberg MC, Klein LW, Agarwal JB, Stets G, Hermann GA, Helfant RH. Quantification of absolute luminal diameter by computer-analyzed digital subtraction angiography: an assessment in human coronary arteries. Circulation 1988; 77: 484-90.

[6] Doriot P.A, Pochon Y, Rasoamanambelo L, Chatelain P, Welz R, Rutishauser W. Densitometry of coronary arteries - An improved physical model. Computers Cardiol. 1985, Computer Society Press, 91-4.

[7] Doriot P-A, Pochon Y, Welz R, Rutishauser W. Non-linearity by densitometric measurements of coronary arteries. In: Heintzen $\mathbf{P H}$ and Bürsch $\mathbf{J}$, eds. Progress in digital cardioangiography, Kluwer Academic Publishers, Dordrecht, The Netherlands, 1988: 173-80.

[8] Rutishauser W Kreislaufanalyse mittels Roentgendensitometrie. Hans Huber Verlag, Bern, 38 pp, 1969.

[9] Bürsch J, Johs R, Heintzen P. Validity of Lambert-Beer's law in roentgendensitometry of contrast material (Urografin) using continuous radiation. In: Heintzen $\mathrm{PH}$, ed. Roentgen-, Cineand Videodensitometry. Georg Thieme Verlag, Stuttgart, 1971: 81-4.

[10] Mol CR, Burridge JM, Morffew AJ. Threedimensional graphic display of X-ray angiographic data. Comput Biomed Res 1986; 19: 47-55.

[11] Wollschläger $H$, Zeiher AM, Lee $P$, Solzbach U, Bonzel $T$, Just $H$. Computed triple orthogonal projections for optimal radiological imaging with biplane isocentric multidirectional $\mathrm{X}$-ray systems. Computers Cardiol 1986, IEEE Computer Society Press: 185-8. 